\title{
Future of Heavy Flavour Physics: Experimental Perspective
}

\author{
Sheldon Stone \\ Physics Department, Syracuse University, Syracuse N. Y., USA, 13244-1130 \\ E-mail: 'stone@phy. syr. edu'
}

Abstract: I discuss what measurements need to be done to search for physics beyond the Standard CKM model, rather than just what studies can be done in the near future. It is also important to accurately measure the CKM matrix elements. Current best estimates for two important elements are: $\left|V_{c b}\right|=0.0381 \pm 0.0021$ and $\left|V_{u b} / V_{c b}\right|=0.085 \pm 0.019$. Finally, future experiments are discussed.

\section{Introduction}

Our goals are to make an exhaustive search for physics beyond the Standard Model and to precisely measure Standard Model parameters. Here we ask what studies need to be done, not just what studies can be done in the near future. Measurements are necessary on $\mathrm{CP}$ violation in $B^{o}$ and $B_{s}$ mesons, $B_{s}$ mixing, rare $b$ decay rates, and mixing, $\mathrm{CP}$ violation and rare decays in the charm sector. These quarks were present in the early Universe. There is a connection between our studies and Cosmology.

\section{The CKM Matrix and CP Viola- tion}

\subsection{The 6 Unitarity Triangles}

The base states of quarks, the mass eigenstates, are mixed to form the weak eigenstates (primed) as described by the Cabibbo-Kobayashi-Maskawa matrix, $V_{C K M}[\underline{1}]$,

$$
\left(\begin{array}{c}
d^{\prime} \\
s^{\prime} \\
b^{\prime}
\end{array}\right)=\left(\begin{array}{lll}
V_{u d} & V_{u s} & V_{u b} \\
V_{c d} & V_{c s} & V_{c b} \\
V_{t d} & V_{t s} & V_{t b}
\end{array}\right)\left(\begin{array}{c}
d \\
s \\
b
\end{array}\right) .
$$

There are 9 complex CKM elements. These 18 numbers can be reduced to 4 independent quantities by applying unitarity constraints and the fact that the phases of the quark wave functions are arbitrary. These 4 remaining numbers are fundamental constants of nature that need to be determined from experiment, like any other fundamental constant such as $\alpha$ or $G$. In the Wolfenstein approximation [2] [ind] $V_{C K M}$ equals:

$$
\begin{array}{ccc}
1-\lambda^{2} / 2 & \lambda & A \lambda^{3}\left(\rho-i \eta\left(1-\lambda^{2} / 2\right)\right) \\
-\lambda & 1-\lambda^{2} / 2-i \eta A^{2} \lambda^{4} & A \lambda^{2}\left(1+i \eta \lambda^{2}\right) \\
A \lambda^{3}(1-\rho-i \eta) & -A \lambda^{2} & 1
\end{array}
$$

This expression is accurate to order $\lambda^{3}$ in the real part and $\lambda^{5}$ in the imaginary part. It is necessary to express the matrix to this order to have a complete formulation of the physics we wish to pursue. The constants $\lambda$ and $A$ have been measured as approximately 0.22 and 0.8 , respectively, using semileptonic $s$ and $b$ decays $[\overline{3} \bar{n}]$. Constraints on $\rho$ and $\eta$ exist from other measurements.

Non-zero $\eta$ allows for CP violation. CP violation thus far has only been seen in the neutral kaon system. If we can find $\mathrm{CP}$ violation in the $B$ system we could see if the CKM model works or perhaps discover new physics that goes beyond the model, if it does not.

The unitarity of the CKM matrix allows us to construct six relationships. These equations may be thought of triangles in the complex plane. They are shown in Figure

All six of these triangles can be constructed knowing four and only four independent angles

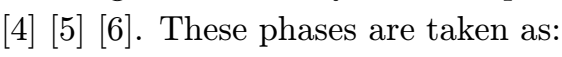

$$
\beta=\arg \left(-\frac{V_{t b} V_{t d}^{*}}{V_{c b} V_{c d}^{*}}\right), \quad \gamma=\arg \left(-\frac{V_{u b}^{*} V_{u d}}{V_{c b}^{*} V_{c d}}\right),
$$




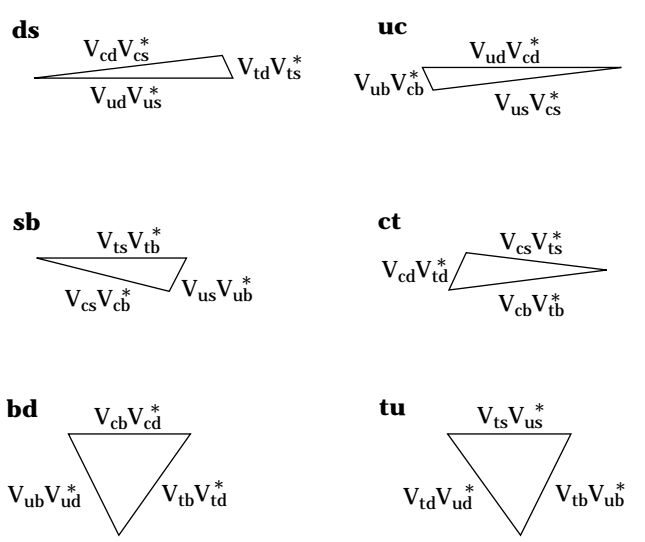

Figure 1: The six CKM triangles. The bold labels, e.g. ds refer to the rows or columns used in the unitarity relationship.

$$
\chi=\arg \left(-\frac{V_{c s}^{*} V_{c b}}{V_{t s}^{*} V_{t b}}\right), \quad \chi^{\prime}=\arg \left(-\frac{V_{u d}^{*} V_{u s}}{V_{c d}^{*} V_{c s}}\right) .
$$

Two of the phases $\beta$ and $\gamma$ are probably large while $\chi$ is estimated to be small $\approx 0.02$, but measurable, while $\chi^{\prime}$ is likely to be one order of magnitude smaller than $\chi$.

In the bd triangle, the one usually considered, the angles are all thought to be relatively large. Since $V_{c d}^{*}=\lambda$, this triangle has sides

$$
\begin{aligned}
& \left|\frac{V_{t d}}{A \lambda^{3}}\right|=\sqrt{(\rho-1)^{2}+\eta^{2}}=\frac{1}{\lambda}\left|\frac{V_{t d}}{V_{t s}}\right| \\
& \left|\frac{V_{u b}}{A \lambda^{3}}\right|=\sqrt{\rho^{2}+\eta^{2}}=\frac{1}{\lambda}\left|\frac{V_{u b}}{V_{c b}}\right| .
\end{aligned}
$$

This CKM triangle is depicted in Figure $\overline{2}$, with constraints from other measurements that will be discussed later.

We know two sides already: the base is defined as unity and the left side is determined by measurements of $\left|V_{u b} / V_{c b}\right|$. The right side can be determined using mixing measurements in the neutral $B$ system. There is, however, a large error due to the uncertainty in $f_{B}$, the $B$-meson decay constant. This error can be greatly reduced by also measuring $B_{s}$ mixing. The figure also shows the angles $\alpha, \beta$, and $\gamma$. Since they form a triangle the "real" $\alpha, \beta$ and $\gamma$ must sum to $180^{\circ}$; therefore measuring any two of these determines the third.

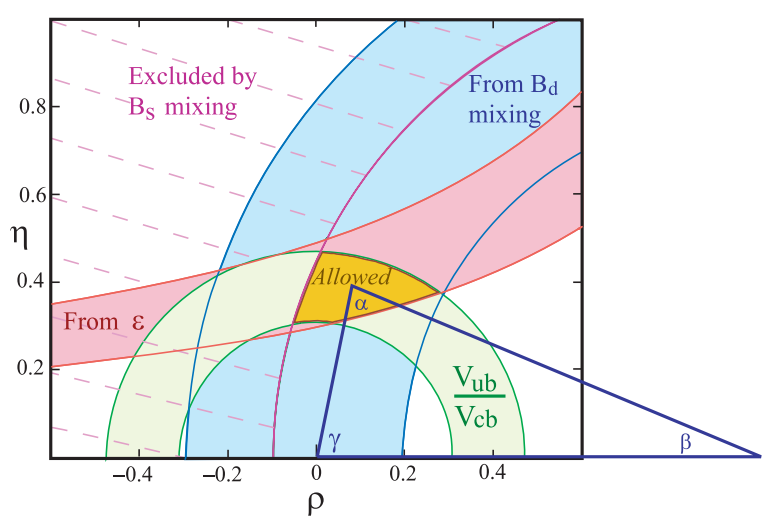

Figure 2: The CKM triangle shown in the $\rho-\eta$ plane. The shaded regions show $\pm 1 \sigma$ contours given by $\left|V_{u b} / V_{c b}\right|$, neutral $B$ mixing, and CP violation in $K_{L}^{o}$ decay $(\epsilon)$. The dashed region is excluded by $B_{s}$ mixing limits. The allowed region is defined by the overlap of the 3 permitted areas, and is where the apex of the CKM triangle sits.

It has been pointed out by Silva and Wolfenstein $\left[\bar{A}_{i}\right]$ that measuring these angles may not be sufficient to detect new physics. For example, suppose there is new physics that arises in $B^{o}-\bar{B}^{o}$ mixing. Let us assign a phase $\theta$ to this new physics. If we then measure $\mathrm{CP}$ violation in $B^{o} \rightarrow J / \psi K_{S}$ and eliminate any Penguin pollution problems in using $B^{o} \rightarrow \pi^{+} \pi^{-}$, then we actually measure $2 \beta^{\prime}=2 \beta+\theta$ and $2 \alpha^{\prime}=2 \alpha-\theta$. So while there is new physics, we miss it, because $2 \beta^{\prime}+2 \alpha^{\prime}=2 \alpha+2 \beta$ and $\alpha^{\prime}+\beta^{\prime}+\gamma=180^{\circ}$.

\subsection{Ambiguities}

In measuring CP phases there are always ambiguities. For example, any determination of $\sin (2 \phi)$, has a four-fold ambiguity; $\phi, \pi / 2-\phi, \pi+\phi$, $3 \pi / 2-\phi$ are all allowed solutions. Often the point of view taken is that we know $\eta$ is a positive quantity and thus we can eliminate two of the four possibilities. However, this would be dangerous in that it could lead to our missing new physics. The only evidence that $\eta$ is positive arises from the measurements of $\epsilon$ and $\epsilon^{\prime}$ and the fact that theoretical calculations give $B_{K}>0$ for $\epsilon$. Even accepting that $K_{L}$ decays give $\eta>0$, it would be foolhardy to miss new physics just because we now assume that $\eta$ must be positive rather than insisting on a clean measurement of the angles that could show a contradiction. 


\subsection{Technique for Measuring $\alpha$}

It is well known that $\sin (2 \beta)$ can be measured without problems caused by Penguin processes using the reaction $B^{o} \rightarrow J / \psi K_{S}$. The simplest reaction that can be used to measure $\sin (2 \alpha)$ is $B^{o} \rightarrow \pi^{+} \pi^{-}$. This reaction can proceed via both the Tree and Penguin diagrams shown in Figure $\overline{\beta_{r}}$
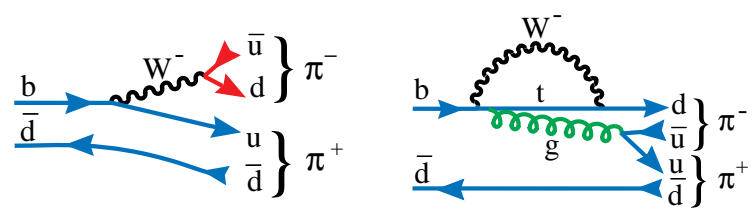

Figure 3: Processes for $B^{o} \rightarrow \pi^{+} \pi^{-}$: Tree (left) and Penguin (right).

Current CLEO results are $\mathcal{B}\left(B^{o} \rightarrow K^{\mp} \pi^{ \pm}\right)=$ $\left(1.88_{-0.26}^{+0.28} \pm 0.13\right) \times 10^{-5}$ and $\mathcal{B}\left(B^{o} \rightarrow \pi^{+} \pi^{-}\right)=$ $\left(0.47_{-0.15}^{+0.18} \pm 0.06\right) \times 10^{-5}[\overline{7} \overline{7}]$, showing a relatively large Penguin amplitude that cannot be ignored. The Penguin contribution to $\pi^{+} \pi^{-}$is roughly half the Tree amplitude. Thus the effect of the Penguin must be determined in order to extract $\alpha$. The only model independent way of doing this was suggested by Gronau and London, but requires the measurement of $B^{\mp} \rightarrow \pi^{\mp} \pi^{o}$ and $B^{o} \rightarrow \pi^{o} \pi^{o}$, the latter being rather daunting.

There is however, a theoretically clean method to determine $\alpha$. The interference between Tree and Penguin diagrams can be exploited by measuring the time dependent $\mathrm{CP}$ violating effects in the decays $B^{o} \rightarrow \rho \pi \rightarrow \pi^{+} \pi^{-} \pi^{o}$ as shown by Snyder and Quinn [8]

The $\rho \pi$ final state has many advantages. First of all, it has been seen with a relatively large rate. The branching ratio for the $\rho^{o} \pi^{+}$final state as measured by CLEO is $(1.5 \pm 0.5 \pm 0.4) \times$ $10^{-5}$, and the rate for the neutral $B$ final state $\rho^{ \pm} \pi^{\mp}$ is $\left(3.5_{-1.0}^{+1.1} \pm 0.5\right) \times 10^{-5}$, while the $\rho^{o} \pi^{o}$ final state is limited at $90 \%$ confidence level to $<5.1 \times 10^{-6}[\overline{9}]$. These measurements are consistent with some theoretical expectations [1 $\left.100_{1}^{1}\right]$. Furthermore, the associated vector-pseudoscalar Penguin decay modes have conquerable or smaller branching ratios. Secondly, since the $\rho$ is spin-1, the $\pi$ spin- 0 and the initial $B$ also spinless, the $\rho$ is fully polarized in the $(1,0)$ configuration, so it decays as $\cos ^{2} \theta$, where $\theta$ is the angle of one of the $\rho$ decay products with the other $\pi$ in the $\rho$ rest frame. This causes the periphery of the Dalitz plot to be heavily populated, especially the corners. A sample Dalitz plot is shown in Figure This kind of distribution is good for maximizing the interferences, which helps minimize the error. Furthermore, little information is lost by excluding the Dalitz plot interior, a good way to reduce backgrounds.

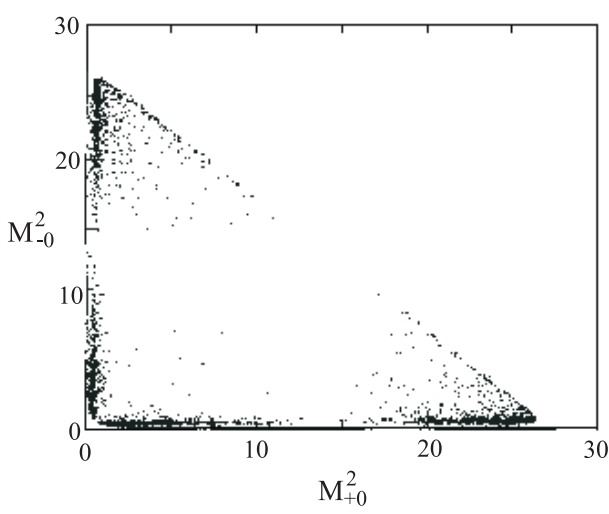

Figure 4: The Dalitz plot for $B^{o} \rightarrow \rho \pi \rightarrow \pi^{+} \pi^{-} \pi^{o}$ from Snyder and Quinn.

To estimate the required number of events Snyder and Quinn preformed an idealized analysis that showed that a background-free, flavortagged sample of 1000 to 2000 events was sufficient. The 1000 event sample usually yields good results for $\alpha$, but sometimes does not resolve the ambiguity. With the 2000 event sample, however, they always succeeded.

This technique not only finds $\sin (2 \alpha)$, it also determines $\cos (2 \alpha)$, thereby removing two of the remaining ambiguities. The final ambiguity can be removed using the $\mathrm{CP}$ asymmetry in $B^{o} \rightarrow$ $\pi^{+} \pi^{-}$and a theoretical assumption [1 i $\left.i_{1}^{1}\right]$.

\subsection{Techniques for Measuring $\gamma$}

In fact, it may be easier to measure $\gamma$ than $\alpha$. There have been at least four methods suggested.

(1) Time dependent flavor tagged analysis of $B_{s} \rightarrow D_{s}^{ \pm} K^{\mp}$. This is a direct model independent measurement $\overline{1} \overline{1} \overline{1}$.

(2) Measure the rate differences between $B^{-} \rightarrow$ $\bar{D}^{o} K^{-}$and $B^{+} \rightarrow D^{o} K^{+}$in two different $D^{o}$ decay modes such as $K^{-} \pi^{+}$and $K^{+} K^{-}$. This method makes use of the interference between 
the tree and doubly-Cabibbo suppressed decays of the $D^{\circ}$, and does not depend on any theoretical modeling [1은.

(3) Rate measurements in two-body $B \rightarrow$ $K \pi$ decays. A cottage industry has developed. However, all methods are model dependent $1 \overline{1} \overline{1}$.

(4) Use U-spin symmetry to relate $B^{o} \rightarrow$ $\pi^{+} \pi^{-}$and $\left.B_{s} \rightarrow K^{+} K^{-}[1] \overline{7}\right]$.

\subsection{Required Measurements Involving $\beta$}

The phase of $B^{o}-\overline{B^{o}}$ mixing will soon be measured by $e^{+} e^{-} b$-factories using the $J / \psi K_{S}$ final state. New physics could be revealed by measuring other final states such as $\phi K_{S}, \eta^{\prime} K_{S}$ or $J / \psi \pi^{o}$.

It is also important to resolve the ambiguities. There are two suggestions on how this may be accomplished. Kayser [1횸] shows that time dependent measurements of the final state $J / \psi K^{\circ}$, where $K^{o} \rightarrow \pi \ell \nu$, give a direct measurement of $\cos (2 \beta)$ and can also be used for CPT tests. Another suggestion is to use the final state $J / \psi K^{* o}$, $K^{* o} \rightarrow K_{S} \pi^{o}$, and to compare with $B_{s} \rightarrow J / \psi \phi$ to extract the sign of the strong interaction phase shift assuming SU(3) symmetry, and thus determine $\cos (2 \beta)$ [1 $19 \overline{9}_{1}^{\prime}$.

\subsection{A Critical Check Using $\chi$}

The angle $\chi$, defined in equation $2-\overline{2} \cdot 3$, can be extracted by measuring the time dependent $\mathrm{CP}$ violating asymmetry in the reaction $B_{s} \rightarrow J / \psi \eta^{(\prime)}$, or if one's detector is incapable of quality photon detection the $J / \psi \phi$ final state can be used. However, there are two vector particles in the final state, making this a state of mixed CP a requiring a complicated time-dependent angular analysis to find $\chi$.

Measurements of the magnitudes of CKM matrix elements all come with theoretical errors. Some of these are hard to estimate; we now try and view realistically how to combine $\mathrm{CP}$ violating phase measurements with the magnitude measurements to best test the Standard Model.

The best measured magnitude is that of $\lambda=$ $\left|V_{u s} / V_{u d}\right|=0.2205 \pm 0.0018$. Silva and Wolfen-

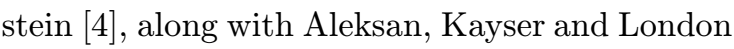
[Fin] show that the Standard Model can be checked in a profound manner by seeing if:

$$
\sin \chi=\left|\frac{V_{u s}}{V_{u d}}\right|^{2} \frac{\sin \beta \sin \gamma}{\sin (\beta+\gamma)} .
$$

Here the precision of the check will be limited initially by the measurement of $\sin \chi$, not of $\lambda$. This check can reveal new physics, even if other checks have not shown any anomalies.

Other relationships to check include:

$$
\begin{aligned}
& \sin \chi=\left|\frac{V_{u b}}{V_{c b}}\right|^{2} \frac{\sin \gamma \sin (\beta+\gamma)}{\sin \beta}, \\
& \sin \chi=\left|\frac{V_{t d}}{V_{t s}}\right|^{2} \frac{\sin \beta \sin (\beta+\gamma)}{\sin \gamma} .
\end{aligned}
$$

The astute reader will have noticed that these two equations lead to the non-trivial constraint:

$$
\sin ^{2} \beta\left|\frac{V_{t d}}{V_{t s}}\right|^{2}=\sin ^{2} \gamma\left|\frac{V_{u b}}{V_{c b}}\right|^{2} .
$$

This contrains these two magnitudes in terms of two of the angles. Note, that it is in principle possible to determine the magnitudes of $\left|V_{u b} / V_{c b}\right|$ and $\left|V_{t d} / V_{t s}\right|$ without model dependent errors by measuring $\beta, \gamma$ and $\chi$ accurately. Alternatively, $\beta, \gamma$ and $\lambda$ can be used to give a much more precise value than is possible at present with direct methods. For example, once $\beta$ and $\gamma$ are known

$$
\left|\frac{V_{u b}}{V_{c b}}\right|^{2}=\lambda^{2} \frac{\sin ^{2} \beta}{\sin ^{2}(\beta+\gamma)} .
$$

\subsection{Other Critical CKM Measurements and Summary}

Magnitudes of the CKM elements are important to measure as precisely as possible. Current measurements of $\left|V_{c b}\right|$ and $\left|V_{u b}\right|$ are discussed in section

It has been predicted that $\Delta \Gamma / \Gamma$ for the $B_{s}$ system is of the order of $10 \%$. This can be determined by measuring the lifetimes in different final states such as $D_{s}^{-} \pi^{+}(\operatorname{mixed} \mathrm{CP}), J / \psi \eta^{\prime}$ (CP $-)$ and $K^{+} K^{-}(\mathrm{CP}+)$. A finite $\Delta \Gamma$ would allow many other interesting measurements of $\mathrm{CP}$ violation $[2 \overline{1}$ i].

Table tities and the suggested decay modes. The necessary detector capabilities include the ability to collect purely hadronic final states, the ability 
to identify charged hadrons, the ability to detect photons with good efficiency and resolution and excellent time resolution required to analyze rapid $B_{s}$ oscillations.

Table 1: Required Measurements for $b$ 's

\begin{tabular}{|l|l|}
\hline $\begin{array}{l}\text { Physics } \\
\text { Quantity }\end{array}$ & Decay Mode \\
\hline $\sin (2 \alpha)$ & $B^{o} \rightarrow \rho \pi \rightarrow \pi^{+} \pi^{-} \pi^{o}$ \\
$\cos (2 \alpha)$ & $B^{o} \rightarrow \rho \pi \rightarrow \pi^{+} \pi^{-} \pi^{o}$ \\
$\operatorname{sign}(\sin (2 \alpha))$ & $B^{o} \rightarrow \rho \pi \& B^{o} \rightarrow \pi^{+} \pi^{-}$ \\
$\sin (\gamma)$ & $B_{s} \rightarrow D_{s}^{ \pm} K^{\mp}$ \\
$\sin (\gamma)$ & $B^{-} \rightarrow \bar{D}^{0} K^{-}$ \\
$\sin (\gamma)$ & $B^{o} \rightarrow \pi^{+} \pi^{-} \& B_{s} \rightarrow K^{+} K^{-}$ \\
$\sin (2 \chi)$ & $B_{s} \rightarrow J / \psi \eta^{\prime}, J / \psi \eta$ \\
$\sin (2 \beta)$ & $B^{o} \rightarrow J / \psi K_{S}$ \\
$\cos (2 \beta)$ & $B^{o} \rightarrow J / \psi K^{o}, K^{o} \rightarrow \pi \ell \nu$ \\
$\cos (2 \beta)$ & $B^{o} \rightarrow J / \psi K^{* o} \& B_{s} \rightarrow J / \psi \phi$ \\
$x_{s}$ & $B_{s} \rightarrow D_{s}^{+} \pi^{-}$ \\
$\Delta \Gamma$ for $B_{s}$ & $B_{s} \rightarrow J / \psi \eta^{\prime}, D_{s}^{+} \pi^{-}, K^{+} K^{-}$ \\
\hline
\end{tabular}

\section{Searches for New Physics}

Because new physics at much larger mass scales can appear in loops, rare process such as $b \rightarrow$ $s \gamma, d \gamma, s \ell^{+} \ell^{-}$and $d \ell^{+} \ell^{-}$have the promise to reveal new physics. Searches in both exclusive and inclusive final states are important.

Charm decays also offer the possibility of finding new physics in the study of either mixing or CP violation as the Standard Model prediction is small. The current experimental measurement of mixing is $r_{D}<5 \times 10^{-3}$, while the SM expectation is $10^{-7}-10^{-6}\left[22_{1}^{\prime}\right]$. For $\mathrm{CP}$ violation the current limits are about $10 \%$, while the expectation is $10^{-3}[\overline{2} \overline{3}]$.

\section{Current Values of $\left|V_{c b}\right|$ and $\left|V_{u b}\right|$, and Allowed Regions in $\rho-\eta$ Plane.}

\subsection{Measurement Of $\left|V_{c b}\right|$ Using $B \rightarrow D^{*} \ell \nu$}

Currently, the most favored technique is to measure the decay rate of $B \rightarrow D^{*} \ell^{-} \bar{\nu}$ at the kinematic point where the $D^{*+}$ is at rest in the $B$ rest frame (this is often referred to as maximum $q^{2}$ or $\omega=1)$. Here, according to Heavy Quark Effective Theory, the theoretical uncertainties are at

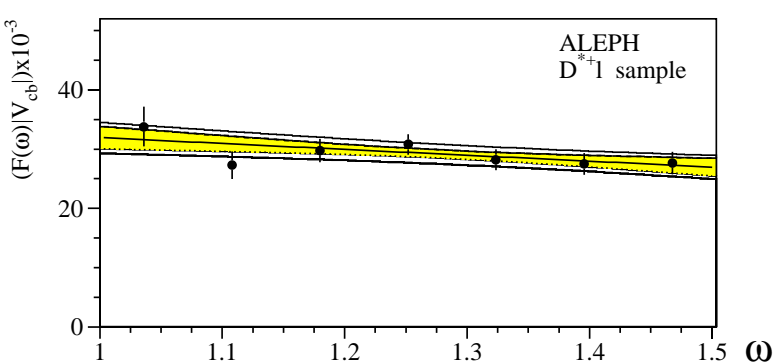

Figure 5: $\quad \bar{B}^{o} \rightarrow D^{+} \ell^{-} \bar{\nu}$ from ALEPH. The data have been fit to a functional form suggested by Caprini et al. The abcissa gives the value of the product $\left|F(\omega) * V_{c b}\right|$.

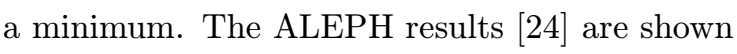
in Figure $\bar{p} \bar{r}$

Table summaries determinations of $\left|V_{c b}\right|$; here, the first error is statistical, the second systematic and the third, an estimate of the theoretical accuracy in predicting the form-factor $F(\omega=1)=0.91 \pm 0.03[25]$. The value and accuracy have been questioned [26]. Hopefully, in the near future a reliable value will be given by lattice QCD without using the quenched approximation [27]. Currently, DELPHI has the smallest error, they detect only the slow $\pi^{+}$from the $D^{*+}$ reconstruct the $D^{o}$ decay. CLEO, however, has only used $1 / 6$ of their current data. The quoted average $\left|V_{c b}\right|=0.0381 \pm 0.0021$ combines the averaged statistical and systematic errors with the theoretical error in quadrature and takes into account the common systematic errors, such as the $D^{*}$ branching ratios.

Table 2: Modern Determinations of $\left|V_{c b}\right|$ using $B \rightarrow$ $D^{*} \ell^{-} \bar{\nu}$ decays at $\omega=1$

\begin{tabular}{|l|c|}
\hline Experiment & $V_{c b}\left(\times 10^{-3}\right)$ \\
\hline ALEPH[24] & $34.4 \pm 1.6 \pm 2.3 \pm 1.4$ \\
DELPHI[28] & $41.2 \pm 1.5 \pm 1.8 \pm 1.4$ \\
OPAL[29.1] & $36.0 \pm 2.1 \pm 2.1 \pm 1.2$ \\
CLEO 30 & $39.4 \pm 2.1 \pm 2.0 \pm 1.4$ \\
\hline Average & $38.1 \pm 2.1$ \\
\hline
\end{tabular}

There are other ways of determining $V_{c b}$. One method based on QCD sum rules uses the operator product expansion and the heavy quark expansion, in terms of the parameters $\alpha_{s}\left(m_{b}\right), \bar{\Lambda}$, and the matrix elements $\lambda_{1}$ and $\lambda_{2}$. The latter 
quantities arise from the differences

$m_{B}-m_{b}=\bar{\Lambda}-\frac{\lambda_{1}+3 \lambda_{2}}{2 m_{b}} m_{B}^{*}-m_{b}=\bar{\Lambda}-\frac{\lambda_{1}-\lambda_{2}}{2 m_{b}}$.

The $B^{*}-B$ mass difference determines $\lambda_{2}=$ $0.12 \mathrm{GeV}^{2}$. The total semileptonic width is then related to these parameters $\left[\bar{B} \overline{1}_{1}^{\prime}\right.$.

CLEO has measured the semileptonic branching ratio using lepton tags as $(10.49 \pm 0.17 \pm 0.43) \%$ and using the world average lifetime for an equal mixture of $B^{o}$ and $B^{-}$mesons of $1.613 \pm 0.020 \mathrm{ps,}$ CLEO finds $\Gamma_{s l}=65.0 \pm 3.0 \mathrm{~ns}^{-1}$.

CLEO then attempts to measure the remaining unknown parameters $\lambda_{1}$ and $\bar{\Lambda}$ by using moments of the either the hadronic mass or the lepton energy [32]. The results are shown in Figure ', $\overline{6}$.'. Here the measurements are shown as bands reflecting the experimental errors. Unfortunately, this preliminary CLEO result shows a contradiction. The overlap of the mass moment bands gives different values than the lepton energy moments! The theoretically favored mass moments give the values $\lambda_{1}=(0.13 \pm 0.01 \pm 0.06)$ $\mathrm{GeV}^{2}$, and $\bar{\Lambda}=(0.33 \pm 0.02 \pm 0.08) \mathrm{GeV}$. The discrepancy between the two methods is serious. It either means that there is something wrong with the CLEO analysis or there is something wrong in the theory, perhaps the breakdown of duality. If the latter is true it would shed doubt on the method used by the LEP experiments to extract $\left|V_{u b}\right|$ using the same theoretical framework.

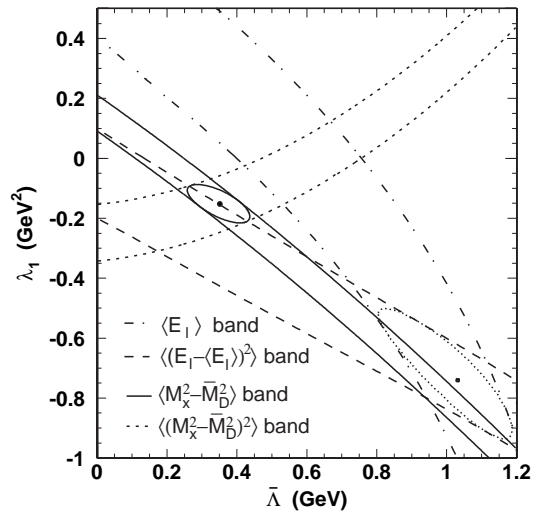

Figure 6: Bands in $\bar{\Lambda}-\lambda_{1}$ space found by CLEO in analyzing first and second moments of hadronic mass squared and lepton energy. The intersections of the two moments for each set determines the two parameters. The $1 \sigma$ error ellipses are shown.

\subsection{Measurement Of $\left|V_{u b}\right|$}

Another important CKM element that can be measured using semileptonic decays is $V_{u b}$. This is a heavy to light quark transition where HQET cannot be used. Unfortunately the theoretical models that can be used to extract a value from the data do not currently give precise predictions.

Three techniques have been used. The first measurement of $V_{u b}$ done by CLEO and subsequently confirmed by ARGUS, used only leptons which were more energetic than those that could come from $b \rightarrow c \ell^{-} \bar{\nu}$ decays [ $\left[\overline{3} \overline{3}_{1}^{\prime}\right]$. These "endpoint leptons" can occur $b \rightarrow c$ background free at the $\Upsilon(4 S)$, because the $B$ 's are almost at rest. Unfortunately, there is only a small fraction of the $b \rightarrow u \ell^{-} \bar{\nu}$ lepton spectrum that can be seen this way, leading to model dependent errors. The models used are either inclusive predictions, sums of exclusive channels, or both [3īin. The average among the models is $\left|V_{u b} / V_{c b}\right|=$ $0.079 \pm 0.006$, without a model dependent error. These models differ by at most $11 \%$, making it tempting to assign a $\pm 6 \%$ error. However, there is no quantitative way of estimating the error.

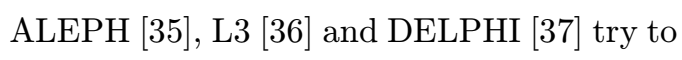
isolate a class of events where the hadron system associated with the lepton is enriched in $b \rightarrow u$ and thus depleted in $b \rightarrow c$. They define a likelihood that hadron tracks come from $b$ decay by using a large number of variables including, vertex information, transverse momentum, not being a kaon etc.. Then they require the hadronic mass to be less than $1.6 \mathrm{GeV}$, which greatly reduces $b \rightarrow c$, since a completely reconstructed $b \rightarrow c$ decay has a mass greater than that of the $D(1.83 \mathrm{GeV})$. They then examine the lepton energy distribution, shown in Figure $\overline{\underline{1}}$, for DELPHI.

The average of all three results as given by the LEP working group [39.1] results in $\left|V_{u b} / V_{c b}\right|=$ $0.106_{-0.020}^{+0.017}$. The results use models [ $\left[\overline{3}_{-}^{\prime \prime}\left[{ }^{2} \overline{6}_{1}^{\prime}\right]\right.$ that assume duality to extract the result. (I have used $\left|V_{c b}\right|=0.0381 \pm 0.0021$.) I have two grave misgivings about this result. First of all the experiments have to understand the level of $b \rightarrow c \ell \nu$ backround to $0.6 \%$. They have not demonstrated that they can do this; there are no experimental checks at this level. Secondly, the theory assumes duality, and there are no successful experimental 


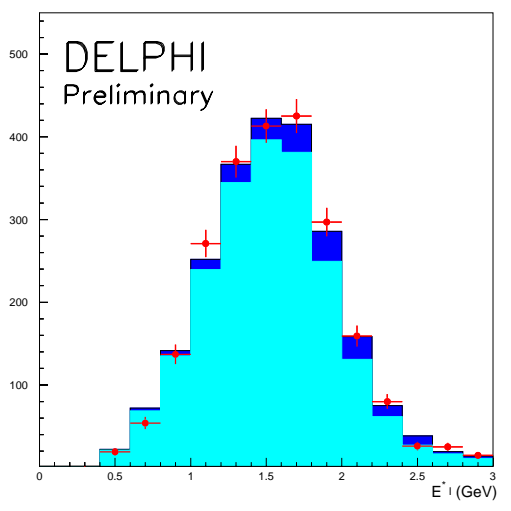

Figure 7: The lepton energy distribution in the $B$ rest frame from DELPHI. The data have been enriched in $b \rightarrow u$ events, and the mass of the recoiling hadronic system is required to be below $1.6 \mathrm{GeV}$. The points indicate data, the light shaded region, the fitted background and the dark shaded region, the fitted $b \rightarrow u \ell \nu$ signal.

checks here either. The one possible check, that of the $b \rightarrow c \ell \nu$ moments has not as yet succeeded. Therefore, I choose not to use these results in my average.

The third method uses exclusive decays. CLEO has measured the decay rates for the exclusive final states $\pi \ell \nu$ and $\rho \ell \nu$ [ $\left.4{ }^{4} 0\right]$ ]. The model of Korner and Schuler (KS) was ruled out by the measured ratio of $\rho / \pi[\overline{3} \overline{4}$. CLEO has recently presented an updated analysis for $\rho \ell \nu$ where they have used several different models to evaluate their efficiencies and extract $V_{u b}$. These theoretical approaches include quark models, light cone sum rules (LCRS), and lattice QCD. The CLEO values are shown in Table $\underline{\underline{3}}$.

Table 3: Values of $\left|V_{u b}\right|$ using $B \rightarrow \rho \ell^{-} \bar{\nu}$ and some theoretical models

\begin{tabular}{|c|c|}
\hline Model & $V_{u b}\left(\times 10^{-3}\right)$ \\
\hline ISGW2[34, & $3.23 \pm 0.14_{-0.29}^{+0.22}$ \\
\hline Beyer/Melnikov [41] & $3.32 \pm 0.15_{-0.30}^{+0.21}$ \\
\hline Wise/Legeti[421] & $2.92 \pm 0.13_{-0.26}^{+0.19}$ \\
\hline $\operatorname{LCSR}\left[43_{-1}^{n}\right]$ & $3.45 \pm 0.15_{-0.31}^{+0.22}$ \\
\hline UKQCD [4] & $3.32 \pm 0.14_{-0.30}^{+0.21}$ \\
\hline
\end{tabular}

The uncertainties in the quark model calculations (first three in the table) are guessed to be $25-50 \%$ in the rate. The Wise/Ligetti model uses charm data and $\mathrm{SU}(3)$ symmetry to reduce the model dependent errors. The other models estimate their errors at about $30 \%$ in the rate, leading to a $15 \%$ error in $\left|V_{u b}\right|$. Note that the models differ by $18 \%$, but it would be incorrect to assume that this spread allows us to take a smaller error. At this time it is prudent to assign a $15 \%$ model dependent error realizing that the errors in the models cannot be averaged. The fact that the models do not differ much allows us to comfortably assign a central value $\left|V_{u b}\right|=\left(3.25 \pm 0.14_{-0.29}^{+0.22} \pm 0.50\right) \times 10^{-3}$, and a derived value $\left|V_{u b} / V_{c b}\right|=0.085_{-0.010}^{+0.008} \pm 0.016$. CLEO could lower this error somewhat if the $\pi \ell \nu$ final state was reanalyzed with LCSR and lattice gauge models.

Only the lattice model predictions of UKQCD are used here. More lattice gauge predictions for the rates in these reactions, at least in some regions of $q^{2}$, are promised soon [4 $\left.4 \overline{4}^{\prime}\right][2 \overline{7} \overline{7}$ ] with better errors. My view is that with experimental checks from measuring form-factors and unquenched lattice gauge models the errors will eventually decrease.

We can use this estimate of $\left|V_{u b} / V_{c b}\right|$ along with other measurements, to get some idea of what the likely values of $\rho$ and $\eta$ are. The $\pm 1 \sigma$ contours shown in Figure ments of CP violation in $K_{L}^{o}$ decay $(\epsilon),\left|V_{u b} / V_{c b}\right|$ and $B^{\circ}$ mixing. Theoretical errors dominate. The limit on $B_{s}$ mixing also restricts the range; its measurement is quite important. Some groups have tried to narrow the "allowed region" by doing maximum liklihood fits, assigning Gaussian errors to the estimated theoretical parameters

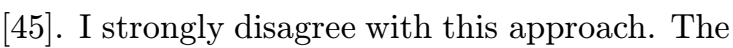
technique of Plaszczynski, shown at this conference [ $[\overline{4} \overline{6}]$, while imprecise, is more justifiable.

\section{Future Experiments}

Lack of space precludes a more through review here. The $e^{+} e^{-}$experiments, BaBar, Belle and CLEO should see CP violation in 2000. The first two in the $J / \psi K_{S}$ final state, while CLEO has a chance of seeing direct $\mathrm{CP}$ violation in rare decays. $\mathrm{CDF}$ and D0 are now scheduled to turn on in 2001. CDF already has seen some evidence for $\mathrm{CP}$ violating effects in $J / \psi K_{S}[\bar{i} \bar{i} \bar{i}]$, and promises 
to measure $B_{s}$ mixing. HERA-B should also turn on in this period.

To overconstrain the CKM matrix and look for new physics all the quantities listed in Table : flavored hadrons, and detectors capable of tolerating large interaction rates and having excellent lifetime resolution, particle identification and $\gamma / \pi^{o}$ detection capabilities. The large $b$ rates, including the $B_{s}$, are available only at hadron colliders. Two dedicated experiments are contemplated, LHC$\mathrm{b}$ which has been approved and $\mathrm{BTeV}$ which is being proposed. Harnew [4] í for_these_two_experiments and ATLAS and_CMS.

in these proceedings. I only add that the $\mathrm{PbWO}_{4}$ EM calorimeter of BTeV should provide important capabilities beyond LHC-b.

[14] M. Gronau and D. Wyler, 'Phys. Lett. B $\mathbf{2} \overline{\mathbf{6}} \overline{1}$ $(172) \overline{19} 1_{1}^{\prime}$

[15] R. Fleischer, and T. Mannel, Phys. Rev. D 57 (1998) 2752 [hep-ph/9704423]; M. Neubert and J. L. Rosner, Phys. Rev. Lett. 81 (1998) 5076 [hep-ph/9809311]; M. Gronau, and J. L. Rosner, Phys. Rev. D 57 (1998) 6843 [hep-ph/9711246]; M. Gronau, and D. Pirjol, [hep-ph/9902482]; M. Gronau and J. L. Rosner, Phys. Rev. D 59 (1999) 113002' [hep-ph/9809384]; J.-M. Gerard and J. Weyers, Eur. Phys. J C7 (1999) 1 [hep$\mathrm{ph} / 9711469]$.

\section{References}

[1] N. Cabibbo, Phys. Rev. Lett. 10, (1963) $531 ; \mathrm{M}$. Kobayashi and K. Maskawa, Prog. Theor. Phys. 49, (1973) 652.

- - [2] L. Wolfenstein,_Phys._Rev_L Lett. 51, (1983)_1945.

[3] S. Stone, "Prospects For B-Physics In The Next Decade," in Techniques and Concepts of HighEnergy Physics IX, ed. by T. Ferbel, NATO ASI Series, Plenum, NY (1996).

[4] J. P. Silva, L. Wolfenstein, Phys. Rev. D 55! '- _ (1997) 5331' [hep-ph/9610208].

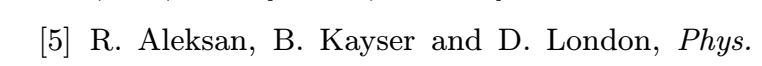

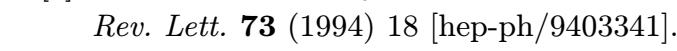

[6] I. I. Bigi and A. I. Sanda, "On the Other Five KM Triangles," [hep-ph/9909479].

[7] Y. Kwon et al. (CLEO), "Study of Charmless Hadronic $B$ Decays into the Final States $K \pi$, $\pi \pi$ and $K K$, with the First Observations of $B^{o} \rightarrow \pi^{+} \pi^{-}$and $B^{o} \rightarrow K^{o} \pi^{o}, "$ Conf $99-14$, [hep-ex/9908039].

[8] A. E. Snyder and H. R. Quinn, Phys. Rev. D 48 (1993) 2139.
[16] A. Falk et al., IPhys. Rev. D $\mathbf{5 7}(1998)+4290$ [hep-th/9712225]; M. Neubert, 'Phys. Lett. _424 (1998) 152 [hep-ph/9712224]; D. Atwood and A. Soni, 'Phys. Rev. D $\mathbf{5 8}(1998) 036005 !$ [hep-ph/9712287]; M. Neubert, J. High Energy Phys. 9902 (1999) 14 [hep-ph/9812396].

[17] R. Fleischer, ${ }_{1}$ Phys. Lett. B 459 (1999) 306 [hep$\mathrm{ph} / 9903456]$.

[18] B. Kayser, "Cascade Mixing and the CPViolating Angle Beta," [hep-ph/9709382]. Previous work in this area was done by Y. Azimov, Phys. Rev. D 42 (1990) 3705.

[19] A. Dighe, I. Dunietz and R. Fleischer, 'Phys.' Lett. B 433 (1998) 147] [hep-ph/9804254].

[20] A. J. Buras, "Theoretical Review of B-physics," in BEAUTY '95 ed. N. Harnew and P. E. Schlein, Nucl. Instru. \& Meth. A368 (1995) 1.

[21] For a recent calculation of $\Delta \Gamma_{s}$, see M. Beneke, G. Buchalla, C. Greub, A. Lenz and U. Nierste, 'Phys. Lett. B 459 (1999) 631' [hep-ph/9808385]. See also I. Dunietz, Phys. Rev. D 52 (1995) 3048.

[22] H. Georgi, ,Phys. Lett. B 297 (1992) 353; T. Ohl et al., 'Nucl. Phys. B 403 (1993) 603. 
[23] F. Buccella et al., 'P F. Buccella et al., "Phys. Lett. B 379 (1996) 249 .

[24] D. Buskulic et al. (ALEPH), Phys. Lett. B 395 (1997) 373.

[25] I. Caprini and M. Neubert, Phys. Lett. B 380(1996) 376; M. Shifman et al., Phys. Rev. D 51(1995) 2217; Erratum-ibid. D 52 (1995) 3149; A. Czarnecki, Phys. Rev. Lett. 76(1996) 4124; T. Mannel, Phys. Rev. D 50 (1994) 428; A. F. Falk and M. Neubert, Phys. Rev. D 47 (1993) 2965 and 2982 .

[26] I. Bigi, "Memo on Extracting $\left|V_{u b}\right|$ and $\left|V_{u b} / V_{c b}\right|$ From Semileptonic $B$ Decays," [hep$\mathrm{ph} / 9907270]$.

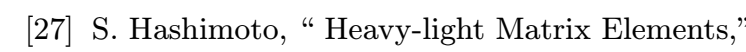
presented at Lattice ${ }^{9} \overline{9}$, the XVIII Int. Symp. on Lattice Field Theory, Pisa, Italy July, 1999, to appear in the proceedings; J.N. Simone et al., "The $B \rightarrow D^{*} \ell \nu$ Form Factor at Zero Recoil" [hep-lat/9910026].

[28] M. Margoni et al., (DELPHI), "Measurement of $V_{c b}$ Using the Identified Charged Pion in $\bar{B}^{o} \rightarrow$ $D^{*+} \ell^{-} \bar{\nu}, "$ DELPHI 98-140, (1998).

[29] K. Ackerstaff et al. (OPAL), Phys. Lett. B 395 (1997) 128.

[30] B. Barish et al. (CLEO), Phys. Rev. D 51 (1995) 1014. The CLEO value has been boosted by $2.6 \%$ to account for the expected negative curvature near $\omega=1$, see S. Stone, Probing the CKM Matrix with b Decays in Proc. of The Albuquerque Meeting, ed. S. Seidel, World Scientific, Singapore (1994) p871.

[31] A. Falk et al., Phys. Rev. D 53 (1996) 2491; ibid D 53 (1996) 6316.

[32] J. Bartelt et al., "Moment Analysis of Inclusive Semileptonic B Meson Decay," submitted to XXIX Int. Conf. on High Energy Physics, Vancouver, Canada (1998) (ICHEP98-1013).

[33] R. Fulton et al. (CLEO), Phys. Rev. Lett. 16 (1990) 64; H. Albrecht et al. (ARGUS), Phys. Lett. B 234 (1990) 409.

[34] N. Isgur and D. Scora (ISGW II), Phys. Rev. D 52 (1995) 2783; N. Isgur, D. Scora, B. Grinstein, and M. B. Wise, Phys. Rev. D 39, (1989) 799; M. Wirbel, B. Stech and M. Bauer Z. Phys. C 29 (1985) 637; M. Bauer and M. Wirbel (WBS), Z. Phys. C 42 (1989) 671; J. G. Korner and G. A. Schuler (KS), Z. Phys. C 38 (1988) 511; ibid, (erratum)C 41 (1989) 690; D. Melikhov, Phys. Rev. D 53 (1996) 2460; G.
Altarelli et al.(ACM), Nucl. Phys. B 208 (1982) 365; C. Ramirez, J. F. Donoghue and G. Burdman (RDB), Phys. Rev. D 41 (1990) 1496.

[35] R. Barate et al. (ALEPH), Eur. Phys. J. C6 (1999) 555.

[36] M. Acciarri et al. (L3), Phys. Lett. B 436 (1998) 174.

[37] M. Battaglia et al. (DELPHI), "Measurement of $\left|V_{u b}\right| /\left|V_{c b}\right|$ with DELPHI at LEP," DELPHI 98-97 CONF 165 submitted to the XXIX Int. Conf. on High Energy Physics, Vancouver, \#241 (1998).

[38] N. Uraltsev et al.. , Eur. Phys. J. C4 (1998) 453 and A. Hoang et al. , Phys. Rev. $\mathbf{D} \mathbf{5 9}$ (1999) 074017, hep-ph/9811239] updated in $\bar{N}$. Uraltsev, "Theoretical Uncertainties in $\Gamma_{s l}(b \rightarrow$ u)," [hep-ph/9905520].

[39] K. Osterberg, "Measurement of $\left|V_{c b}\right|$ and $\left|V_{u b}\right|$ at LEP," presented at EPS HEP-99, Tampere, Findland, 1999, to appear in proceedings. For updates see http://home.cern.ch/a/abbaneo/www/vub.html .

[40] J. P. Alexander et al. (CLEO), Phys. Rev. Lett. 77 (1996) 5000.

[41] M. Beyer and D. Melikhov, Phys. Lett. B 436 (1998) 344.

[42] Z. Legeti and M. B. Wise, Phys. Rev. D 53 (1996) 4937.

[43] P. Ball and V. M. Braun, Phys. Rev. D 58 (1998) 094016.

[44] L. Lellouch, "Exclusive semileptonic B decays: dispersive bounds and lattice results," in these proceedings.

[45] F. Parodi, P. Roudeau, A. Stocchi, "Constraints on the parameters of the CKM matrix by End 1998," [hep-ex/9903063]; S. Mele, Phys. Rev. D 59 (1999) 113011.

[46] S. Plaszczynski, "Overall determination of the CKM matrix," in these proceedings.

[47] D. Bortoletto, "Measurement of $\sin (2 \beta)$," in these proceedings; T. Affolder et al. (CDF), "A Measurement of $\sin 2 \beta$ from $B \rightarrow J / \psi K_{S}^{0}$ with the CDF detector," [hep-ex/9909003].

[48] N. Harnew, "Prospects for LHC-b, BTeV, ATLAS, CMS," in these proceedings. For more information on $\mathrm{BTeV}$ see http://wwwbtev.fnal.gov/btev.html, for LHCb see http://lhcb.cern.ch/ . 